Horizons philosophiques

\title{
La vulgarisation philosophique : pour ou contre?
}

\section{Ariane Poulantzas}

Volume 8, numéro 2, printemps 1998

Défense et illustration de la vulgarisation philosophique

URI : https://id.erudit.org/iderudit/801073ar

DOI : https://doi.org/10.7202/801073ar

Aller au sommaire du numéro

\section{Éditeur(s)}

Collège Édouard-Montpetit

\section{ISSN}

1181-9227 (imprimé)

1920-2954 (numérique)

Découvrir la revue

Citer cet article

Poulantzas, A. (1998). La vulgarisation philosophique : pour ou contre? Horizons philosophiques, 8(2), 41-46. https://doi.org/10.7202/801073ar d'utilisation que vous pouvez consulter en ligne.

https://apropos.erudit.org/fr/usagers/politique-dutilisation/ 


\section{LA VULGARISATION PHILOSOPHIQUE : POUR OU CONTRE?}

"La vulgarisation philosophique», voilà un sujet d'actualité, un sujet qui se pose, qui s'impose, un sujet qui divise, qui suscite des passions. La question qui lui est inhérente est celle de sa légitimité. II ne s'agit pas tant de savoir si la vulgarisation philosophique est possible que de savoir si elle est souhaitable. Non pas : comment peut-on vulgariser la philosophie? $\mathrm{Ni}$ même : peut-on la vulgariser? Mais bien : doit-on le faire? A-ton le droit de vulgariser la philosophie sans déchoir de son statut de philosophe?

Mais ce constat une fois fait, il s'agit d'en comprendre les causes : cette réserve, sinon cette répugnance, est-elle due à l'exercice philosophique lui-même (il y aurait dans toute philosophie une impossibilité intrinsèque à être accessible à tous; la philosophie serait structurellement élitiste), ou est-elle due à une attitude commune à la plupart des philosophes qui, loin de refléter la nature intime de la philosophie, reflète plutôt la place conjoncturelle qu'elle occupe aujourd'hui dans notre société?

On pourrait tenter de trancher cette alternative en analysant l'attitude des philosophes au cours des siècles. Mais l'éclairage apporté ne nous permettrait pas pour autant de répondre rigoureusement à la question. En effet, quand bien même on retrouverait, de tout temps, des réticences de la part des philosophes à la diffusion de leur discipline, on ne pourrait en conclure l'impossibilité pour le large public à comprendre la philosophie.

Admettons que la philosophie soit intrinsèquement destinée à un public étroit, qu'elle ne soit pas, en droit, vulgarisable. Quelles pourraient en être les raisons? Lequel de ses caractères pourrait en interdire l'accès à un large public?

Sa difficulté, son aridité, sa technicité? Sans doute. Mais alors pourquoi les scientifiques n'ont, en général, pas de réti- 
cence à tenter d'expliquer leurs découvertes au grand public (qu'ils y arrivent plus ou moins bien est une autre question). Lorsque l'on parle de vulgarisation scientifique, on ne se demande pas si elle est légitime mais si elle est possible; il va de soi que le public a le droit, voire le devoir, de se tenir au courant des principales avancées scientifiques. Certes cette diffusion de la science, ou plutôt des sciences, n'est pas exempte de difficultés : comment faire l'économie de la technicité, comment simplifier sans trahir? Ces questions sont incontournables. Vulgariser, c'est nécessairement perdre en rigueur et en substance. Mais ces difficultés, voire ces limites de la vulgarisation scientifique n'invalident en rien la dignité de l'entreprise. Pourquoi n'en est-il pas de même pour la philosophie? La difficulté, la technicité, l'aridité seraient-elles encore plus fondamentales qu'en science? Non, bien sûr. Mais alors?

La difficulté formelle d'une discipline ne semble pas constituer un obstacle majeur, sinon à la réussite de sa vulgarisation du moins à la tentative de cette dernière. La technicité d'une discipline peut empêcher qu'elle soit, de fait, vulgarisée mais non qu'elle soit, en droit, vulgarisable.

Si la réticence des philosophes à vulgariser leur discipline ne tient pas à la forme de cette dernière, tient-elle à son objet? L'objet de la philosophie est-il réservé à un public restreint? Les questions posées par la philosophie sont-elles absolument et nécessairement étrangères à celles du peuple?

Sans prétendre, ici, définir la philosophie, on peut tout de même souligner que son objet, qu'il soit éthique, politique ou même métaphysique, peut faire écho aux interrogations de tout un chacun. Comment bien vivre? Comment être heureux? Qu'est-ce que le bien? Comment vivre ensemble? Comment accepter la mort? Qu'est-ce que connaître? Qu'est-ce que penser? Qu'est-ce qu'aimer?

Ces questions semblent plus proches des préoccupations communes que la plupart des problèmes scientifiques. Dès lors, on ne peut justifier le refus de s'essayer à une tentative de vulgarisation philosophique par le désintérêt public. D'ailleurs, 
l'actuelle demande de philosophie est un démenti criant de ce type d'arguments. La situation est pour le moins étrange : le public est en demande de vulgarisation philosophique et les professionnels (les vrais) s'y refusent.

Si ni la forme aride de la philosophie, ni ses objets d'investigation, n'invalident la possibilité d'une transmission au grand public, comment expliquer la réticence, la méfiance des professionnels?

Et si ce qui faisait peur aux philosophes était précisément la proximité de leurs interrogations avec celles du commun des mortels? la philosophie se pose les mêmes questions que tout un chacun, mais elle se les pose autrement. Dès lors, le risque est celui de l'assimilation abusive. Entre la science et la pensée commune, pas de confusion possible, pas de risque pour le scientifique de voir sa pensée assimilée à une pensée néophyte. Pour le philosophe, au contraire, parce que les questions qui constituent son univers théorique sont comme le miroir des interrogations communes, le risque d'une assimilation abusive se profile toujours. La philosophie naît d'une interrogation commune mais la dépasse : et c'est ce dépassement, ce détour par le concept, qui, difficilement accepté par le public, menace la spécificité philosophique. Le scientifique n'a pas à démontrer que son exercice demande une compétence particulière; son objet est d'emblée étranger. Le philosophe, au contraire, doit sans cesse lutter contre l'assimilation entre son exercice et une certaine pensée commune; fondamentalement, leurs objets se rejoignent.

Ce qui empêche la vulgarisation philosophique ce n'est donc pas son étrangeté radicale d'avec la pensée commune mais, au contraire, sa grande proximité.

Mais cette difficulté est-elle indépassable? La philosophie est-elle nécessairement menacée par la pensée commune, par ce faux double? Peut-on imaginer une vulgarisation philosophique réussie, c'est-à-dire une expression accessible de la philosophie qui, pour autant, ne perde pas sa spécificité? Autrement dit, peut-on faire accepter au public le détour par un 
certain langage technique? On le voit, l'obstacle n'est pas tant théorique que psychologique : la difficulté n'est pas de faire comprendre les concepts et les méandres de l'analyse philosophique mais de faire admettre que le recours à ces derniers est nécessaire.

Dès lors, on peut comprendre les réticences des philosophes face à la demande du public : s'agit-il d'un authentique désir de philosopher? Que demande véritablement le public lorsqu'il réclame de la philosophie? Ne retient-il pas de la philosophie seulement les questions qu'elle pose, laissant de côté la manière tout à fait spécifique dont elle tente d'y répondre? Dès lors, la réserve du philosophe n'est-elle pas fondée?

II semble ici que le terme "public» regroupe des réalités beaucoup trop disparates et que cette assimilation discrédite à tort certaines demandes philosophiques tout à fait authentiques. Mais comment faire le tri? Comment préjuger de la nature du désir "philosophique» de tel ou tel?

Cette assimilation préjudiciable entre plusieurs demandes hétérogènes est parfaitement illustrée par les fameux "cafés philosophiques». En effet, ce qui y saute aux yeux, c'est l'inauthenticité du désir philosophique de certains. Ce qui bien sûr n'implique pas que leur présence ne relève d'aucun désir authentique (désir de distractions, de paroles, de rencontres, de sociabilité). Ces désirs sont tout à fait respectables mais ils n'en sont pas philosophiques pour autant. Dès lors, l'animateur à se faire trop pédagogue sombre souvent dans la démagogie. Mais, d'une certaine façon, où est le mal si le public est satisfait et s'il l'est d'autant plus qu'il s'imagine faire de la philosophie?

Encore une fois, ce que l'on peut reprocher à ces pratiques, et surtout au fait qu'elles s'intitulent "philosophiques», c'est le discrédit qu'elles portent sur des demandes réellement philosophiques bien que provenant de néophytes. Dans cette histoire, ce sont ces derniers qui pâtissent; ce sont eux qui demeurent sur leur faim. Le seul moyen qu'ils ont de satisfaire leur demande est de suivre les cours à l'université. Mais, qui sait que les cours sont ouverts à tous et qui se risque à 
s'asseoir au milieu d'étudiants souvent beaucoup plus jeunes, inscrits dans un cursus strict et qui, pour la plupart se destinent eux-mêmes à l'enseignement? De surcroît, le seul mode sérieux de transmission de la philosophie est-il le cours magistral? Ne peut-on pas imaginer d'autres modes qui laisseraient plus de place aux interrogations de chacun sans toutefois tomber dans les travers du "café du commerce»? Ne peut-on pas dépasser l'alternative : cafés "philo-démago", enseignement universitaire? En d'autres termes, la demande philosophique peut-elle trouver une réponse adéquate? Le philosophe peut-il répondre à la demande qui lui est faite?

Malheureusement, le philosophe n'accède que rarement à la demande du public, et ceci quelle que soit sa bonne volonté. La demande du public est souvent si forte que la réponse du philosophe reste toujours en deçà. La philosophie et le philosophe qui en est le représentant bénéficient et pâtissent en même temps de leur réputation. La philosophie n'est pas une discipline comme les autres; elle est méprisée ou admirée (et ceci avant même d'être connue). Sa réputation la précède toujours. Dès lors, le philosophe a toujours à lutter contre ces préjugés, qu'ils soient négatifs ou positifs. Et la lutte la plus difficile est sans doute celle qui consiste à combattre les préjugés positifs démesurés qui auréolent à tort la philosophie; non, la philosophie n'a pas de recette toute faite à délivrer; non, elle n'est pas une thérapie; non, elle ne livre pas le sens de la vie! Le malentendu semble de mise.

Encore une fois, le terme "philosophie" n'a souvent pas le même sens pour le public et pour le professionnel. Or, il cristallise trop de passions pour que l'on puisse s'accorder. Tout le monde se réclame de la philosophie, de la vraie philosophie (de la spontanée ou de la laborieuse, de celle "de la vie" ou de l'«analytique», selon). Tout le monde s'en réclame sauf ceux qui mettent leur point d'honneur à la rejeter.

Le malentendu est de mise et il semble indépassable. II ne s'agit pas uniquement d'une querelle de mots. Définir la philosophie, c'est toujours prétendre en donner une définition réelle et 
pas seulement nominale. Dès lors, ce n'est pas seulement sur le mot que l'on ne s'accorde pas mais réellement sur la chose.

Or, on le sait, définir la philosophie est déjà une tâche philosophique. Dès lors, toute tentative de vulgarisation ne devrait-elle pas s'inscrire d'emblée dans cette interrogation? Ceci permettrait, sinon de dissiper le malentendu, du moins de le circonscrire.

Ariane Poulantzas 